\title{
Front Matter: Volume 10016
}

, "Front Matter: Volume 10016," Proc. SPIE 10016, High-Power Lasers and Applications VIII, 1001601 (27 December 2016); doi: 10.1117/12.2267239

SPIE. Event: SPIE/COS Photonics Asia, 2016, Beijing, China 


\title{
PROCEEDINGS OF SPIE
}

\section{High-Power Lasers and Applications VIII}

\author{
Ruxin Li \\ Upendra N. Singh \\ Robert F. Walter \\ Editors
}

\section{2-14 October 2016 Beijing, China}

Sponsored by

SPIE

COS-Chinese Optical Society

\section{Cooperating Organizations}

Tsinghua University (China) • Peking University (China) • University of Science and Technology of China (China) • Zhejiang University (China) • Tianjin University (China) • Beijing Institute of Technology (China) • Beijing University of Posts and Telecommunications (China) • Nankai University (China) • Changchun University of Science and Technology (China) • University of Shanghai for Science and Technology (China) • Capital Normal University (China) - Huazhong University of Science and Technology (China) • Beijing Jiaotong University (China) • Shanghai Institute of Optics and Fine Mechanics (China) - Changchun Institute of Optics and Fine Mechanics (China) • Institute of Semiconductors (China) • Institute of Optics and Electronics (China) • Institute of Physics (China) • Shanghai Institute of Technical Physics (China) • China Instrument and Control Society (China) • Optoelectronics Technology Committee, COS (China) • SPIE National Committee in China (China) • Optical Society of Japan (Japan) • Optical Society of Korea (Korea, Republic of) - The Australian Optical Society (Australia) • Optics and Photonics Society of Singapore (Singapore) • European Optical Society

\section{Supporting Organizations}

CAST_China Association for Science and Technology (China)

NSFC-National Nature Science Foundation (China)

Published by

SPIE

\section{Volume 10016}


The papers in this volume were part of the technical conference cited on the cover and title page. Papers were selected and subject to review by the editors and conference program committee. Some conference presentations may not be available for publication. Additional papers and presentation recordings may be available online in the SPIE Digital Library at SPIEDigitallibrary.org.

The papers reflect the work and thoughts of the authors and are published herein as submitted. The publisher is not responsible for the validity of the information or for any outcomes resulting from reliance thereon.

Please use the following format to cite material from these proceedings:

Author(s), "Title of Paper," in High-Power Lasers and Applications VIII, edited by Ruxin Li,

Upendra N. Singh, Robert F. Walter, Proceedings of SPIE Vol. 10016 (SPIE, Bellingham, WA, 2016) Seven-digit Article CID Number.

ISSN: 0277-786X

ISSN: 1996-756X (electronic)

ISBN: 9781510604513

ISBN: 9781510604520 (electronic)

Published by

SPIE

P.O. Box 10, Bellingham, Washington 98227-0010 USA

Telephone +1 3606763290 (Pacific Time) · Fax +1 3606471445

SPIE.org

Copyright @ 2016, Society of Photo-Optical Instrumentation Engineers.

Copying of material in this book for internal or personal use, or for the internal or personal use of specific clients, beyond the fair use provisions granted by the U.S. Copyright Law is authorized by SPIE subject to payment of copying fees. The Transactional Reporting Service base fee for this volume is $\$ 18.00$ per article (or portion thereof), which should be paid directly to the Copyright Clearance Center (CCC), 222 Rosewood Drive, Danvers, MA 01923. Payment may also be made electronically through CCC Online at copyright.com. Other copying for republication, resale, advertising or promotion, or any form of systematic or multiple reproduction of any material in this book is prohibited except with permission in writing from the publisher. The CCC fee code is 0277-786X/16/\$18.00.

Printed in the United States of America.

Publication of record for individual papers is online in the SPIE Digital Library.

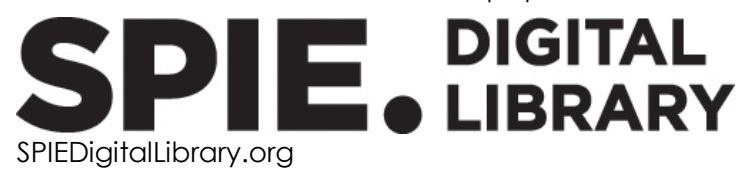

Paper Numbering: Proceedings of SPIE follow an e-First publication model. A unique citation identifier (CID) number is assigned to each article at the time of publication. Utilization of CIDs allows articles to be fully citable as soon as they are published online, and connects the same identifier to all online and print versions of the publication. SPIE uses a seven-digit CID article numbering system structured as follows:

- The first five digits correspond to the SPIE volume number.

- The last two digits indicate publication order within the volume using a Base 36 numbering system employing both numerals and letters. These two-number sets start with 00, 01, 02, 03, 04, $05,06,07,08,09,0 A, 0 B \ldots$ OZ, followed by 10-1Z, 20-2Z, etc. The CID Number appears on each page of the manuscript. 


\title{
Contents
}

\author{
vii Authors \\ xi Symposium Committees \\ xiii Conference Committee
}

\section{SESSION 1 LASER APPLICATIONS}

1001603 High power DUV lasers for material processing [100166-2]

1001606 Research of silicon solar cells' performance after being irradiated by high power laser [100166-5]

\section{SESSION 2 OTHER LASERS AND APPLICATIONS}

1001609 Low threshold and high efficiency solar-pumped laser with Fresnel lens and a grooved Nd:YAG rod [100166-8]

10016 OC Enhanced visible supercontinuum generation in seven-core photonic crystal fiber [100166-11]

\section{SESSION 3 HIGH POWER LASERS I}

10016 OF Edge-pumped multi-slab amplifier for inertial fusion energy (IFE) [100166-15]

$100160 G$ High power far-infrared optical parametric oscillator with high beam quality (Invited Paper) [100166-17]

\section{SESSION 4 HIGH POWER LASERS II}

10016 ol Large aperture laser beam alignment system based on far field sampling technique [100166-16]

10016 J Correction of the phase distortion for high power laser by freeform shape mirror [100166-20]

$100160 \mathrm{~K}$ Investigation on the formation of intense fringe near nonlinear medium slab in nonlinear imaging [100166-21] 
10016 OM Recent development on high-power tandem-pumped fiber laser (Invited Paper) [100166-23]

10016 ON 1-MW peak power, 574-kHz repetition rate picosecond pulses at $515 \mathrm{~nm}$ from a frequencydoubled fiber amplifier [100166-25]

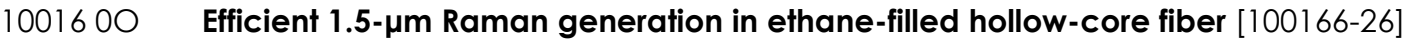

10016 OP Systematic analysis of nanosecond pulse amplification in Ytterbium-doped double-clad fiber amplifiers by considering inelastic scatterings and different operation regimes [100166-27]

$100160 Q \quad T h e o r e t i c a l$ and experimental analysis of double-pass ytterbium-doped fiber amplifier [100166-28]

10016 OR Passive harmonic mode locking in a monolayer graphene-based long cavity fiber laser with high pulse energy [100166-29]

10016 OS All-fiberized single-frequency polarization-maintained fiber amplifier with record power [100166-30]

10016 OT Linear inner cladding fiber amplifier suppressing mode instability [100166-31]

10016 OU Phase modulation signals optimization automatically for suppression of stimulated Brillouin scattering [100166-32]

\section{SESSION $6 \quad$ HIGH FIELD LASER PHYSICS}

10016 OW Interaction of high power laser beams with plasma in ICF hohlraum using the FDTD method [100166-34]

10016 OY Studies on high-quality electron beams and tunable $x$-ray sources produced by laser wakefield accelerators (Invited Paper) [100166-37]

\section{SESSION 7 ULTRAFAST LASERS}

1001610 Measurement and compensation schemes for the pulse front distortion of ultra-intensity ultra-short laser pulses [100166-39]

$1001612 \quad$ 58W LD side-pump Nd:YAG picosecond laser system at $1 \mathrm{KHz}$ with double length of regenerative cavity [100166-41]

1001614 Mode-locked femtosecond all polarization-maintaining erbium-doped dispersion managed fiber laser based on a nonlinear amplifying loop mirror [100166-43]

1001615 Stable linear SESAM femtosecond fiber laser by use of faraday rotator mirrors [100166-44] 
1001617 The influence of combined guiding effect on the beam quality in MOPA Nd:YVO 4 lasers [100166-13]

1001618 63W output tandem-pumped thulium-doped silica fiber laser at $1980 \mathrm{~nm}$ [100166-24]

1001619 Research on the energy coupling coefficient of 45\# steel and $\mathbf{3 0 4}$ stainless steel under 3.8um CW laser irradiation [100166-36]

$100161 \mathrm{~A}$ The outgassing characteristic research of the silicone rubber in high power laser system [100166-46]

$100161 \mathrm{~B} \quad$ Numerical modeling of three-level system in ytterbium-doped photonic crystal fiber laser [100166-47]

$100161 \mathrm{C}$ Structural-optical integrated analysis on the large aperture mirror with active mounting [100166-48]

10016 1D Design and fabrication of transmission gratings with high diffraction efficiency for pulse compression [100166-49]

$100161 \mathrm{~F} \quad$ Coherent combining efficiency improvement based on a focused conformal projection fiber laser array [100166-51]

$100161 \mathrm{G}$ Longitudinally excited $\mathrm{CO}_{2}$ laser with short laser pulse operating at high repetition rate [100166-52]

10016 1H Vortex beam based more stable annular laser guide star [100166-53]

1001611 Effect of nonlinearity saturation on hot-image formation in cascaded saturable nonlinear medium slabs [100166-54]

$100161 \mathrm{~J} \mathrm{Formation} \mathrm{of} \mathrm{hot} \mathrm{image} \mathrm{in} \mathrm{an} \mathrm{intense} \mathrm{laser} \mathrm{beam} \mathrm{through} \mathrm{a} \mathrm{saturable} \mathrm{nonlinear} \mathrm{medium}$ slab [100166-55]

10016 1K Design of high efficiency, broad bandwidth unpolarized transmission grating for femtosecond laser [100166-56]

$100161 \mathrm{M}$ Experimental investigation of thermally induced core laser leakage in large mode area single trench fiber [100166-58]

$100161 \mathrm{~N} \quad$ Longitudinally excited $\mathrm{CO}_{2}$ laser with multiple laser tubes [100166-59]

$100161 \mathrm{P}$ Thermal effects of the gradient doping gain fiber in all-fiber MOPA [100166-61]

$100161 Q \quad$ Theoretical study of transverse mode selection in laser resonator with volume Bragg gratings [100166-62]

10016 1R Output characteristics of diode-pumped Yb:YAG mode-locked laser with a dual-core fiber [100166-63] 
$100161 \mathrm{~T}$ Research on polarization vector characteristics in a microfiber-based graphene fiber laser [100166-65]

$100161 \mathrm{U}$ Formation of noise-like square-wave pulses in a microfiber based topological insulator fiber laser [100166-66]

10016 IV Experimental investigation of high energy noise-like pulses from a long cavity erbiumdoped fiber laser [100166-67]

10016 IW Dynamic measurement of reflectance/emissivity in mid-infrared band [100166-68]

$100161 \mathrm{X}$ Simulation of wavefront optimization with volume Bragg grating in photothermorefractive glass [100166-69]

10016 IY Laser-induced damage on fused silica with photo-acoustic method [100166-70]

1001612 Study and optimization of key parameters of a laser ablation ion mobility spectrometer [100166-71]

1001620 Influence of soft bonding layer material viscoplasticity on thermal lens and aspherical aberration of high-power thin disk laser [100166-73]

1001621 The design of thin disk laser multi-pass amplifier [100166-74]

1001622 New type of fiber mode stripper with quartz tubes [100166-75] 


\section{Authors}

Numbers in the index correspond to the last two digits of the seven-digit citation identifier (CID) article numbering system used in Proceedings of SPIE. The first five digits reflect the volume number. Base 36 numbering is employed for the last two digits and indicates the order of articles within the volume. Numbers start with 00, 01, 02, 03, 04, 05, 06, 07, 08, 09, OA, OB...0Z, followed by 10-1Z, 20-2Z, etc.

\author{
Abdollahi, M., OP, 15 \\ Akitsu, Tetsuya, 1G, 1N \\ Bagheri Harouni, M., OP \\ Bai, Yang, $\mathrm{ON}$ \\ Bu, Fan, 18 \\ Chang, Zenghu, ID \\ Chen, Liezun, 1I, $1 \mathrm{~J}$ \\ Chen, Meng, 12 \\ Chen, Min, OY \\ Chen, Minsun, 06, 19, 1W \\ Chen, Sheng-Ping, OC \\ Chen, Tao, 1B \\ Chen, Xinrong, 1D, $1 \mathrm{~K}$ \\ Chen, Yongqian, 22 \\ Chen, Yubin, 00 \\ Cui, Wenda, $1 \mathrm{H}$ \\ Cui, Xudong, OF \\ Dai, Jian, 14 \\ Dai, Neng-li, 18 \\ Dai, Tong-Yu, OG \\ Dai, Yitang, 14 \\ Dai, Zhiping, 1I, $1 \mathrm{~J}$ \\ Dong, Meng, IA \\ Du, Daiyan, $0 Q$ \\ Duan, Xiao-Ming, OG \\ Fakhari, M., OP \\ Feng, Yali, $1 \mathrm{U}$ \\ Feng, Yufan, 20 \\ Fu, Zhaohui, $1 \mathrm{~A}$ \\ Fujimoto, Junichi, 03 \\ Gao, Fan, 1Q, 1X \\ Gao, Fengbin, 09 \\ Gao, Jiapeng, 20 \\ Gholami, A., 15 \\ Gong, L., Ol \\ Gu, Bo, 00 \\ Gu, Shaoyi, 1M \\ Guan, Zhe, 09 \\ Guo, Shaofeng, $1 \mathrm{M}$ \\ Guoyu, Heyang, IV \\ Han, Huiyun, 1T, $1 \mathrm{U}$ \\ Han, Kai, IP \\ Han, Mengmeng, 1T, $1 U$ \\ $\mathrm{He}$, Xiaoying, $\mathrm{OR}$ \\ $\mathrm{He}$, Yulong, $1 \mathrm{H}$ \\ Hekmat, M. J., OP, 15 \\ Hou, Jing, OC \\ Hu, Jing, $1 Q$ \\ $\mathrm{Hu}$, Yonghua, OK
}

Hu, Zuyuan, 1D

Huang, Liangjin, $1 M$

Huang, Long, OQ, OS

Huang, Qitai, OJ

Huang, Zhihua, OT

Jiang, Hou-man, IW

Jiang, Min, OU

Jiang, Xinying, OF

Jiang, Zongfu, $1 \mathrm{M}$

Jing, Feng, OT

Jitsuno, Takahisa, 1G, IN

Kakizaki, Kouji, 03

Kanani, M., OP, 15

Kang, J., $\mathrm{Ol}$

$\mathrm{Ke}$, Jieyao, 09

$\mathrm{Ke}, \mathrm{Kai}, \mathrm{IY}$

Kobayashi, Masakazu, 03

Kong, Lingchao, $1 M$

Leng, Jinyong, $0 M, 1 M$

Leng, Yuxin, 10

Li, Chaoming, 1D, IK

Li, G., 12

Li, Hai-qing, 18

$\mathrm{Li}$, Jianan, $1 \mathrm{Z}$

Li, Jianhui, $1 \mathrm{G}$

Li, Jin-yan, 18

Li, Kexuan, IV

Li, Lei, OS, 1H

Li, Lin, ID

Li, Min, OF

Li, Mingzhong, OF

Li, Qiurui, ON

Li, Ruixian, OS

Li, Wenkai, 10

Li, Xiaoyang, 1D

Li, Xingliang, 1T, IU

Li, Yanjie, 06

Li, Yunfei, 06

Li, Zebiao, OT

Lin, Honghuan, OT

Lin, Zhili, OW

Ling, Xiaohui, 11, $1 \mathrm{~J}$

Liu, D. Z., Ol

Liu, Jingmin, 1T, $1 \mathrm{U}$

Liu, Qiang, 17

Liv, Tong, OC

Liu, Zhigang, $1 \mathrm{C}$

Long, Ming Liang, 12

Lu, Jun, 10 
Lu, Qisheng, 00

Lu, Shizhuan, 11, $1 \mathrm{~J}$

Luo, Ji, OY

Luo, Ruiyao, $1 \mathrm{H}$

LV, Haibing, 1 A

Ma, Pengfei, OS

$\mathrm{Ma}$, Yanxing, IF

Malek Mohammadi, S., 15

Matsunaga, Takashi, 03

Mimura, Toshio, 03

Mizoguchi, Hakaru, 03

$\mathrm{Ni}, \mathrm{Kai}, 1 \mathrm{Z}$

Ning, $\mathrm{Yu}, 1 \mathrm{H}$

Noormohammadi, H., OP, 15

Oizumi, Hiroaki, 03

Omoomi, M., 15

Oyang, X.P., Ol

Peng, Jing-gang, 18

Peng, Xue, OK

Qi, Lijun, 22

Qi, Xue, OC

Qian, Chuan-Peng, $0 \mathrm{G}$

Qian, Xiang, 12

Qiu, Yaqiong, OK

Qiu, Yuli, 22

Ran, Yang, OU

Ren, Jianfeng, OJ

Ren, Zhiyuan, $1 \mathrm{C}$

Shahriari, N., OP, 15

Shang, Ce, $1 U$

Shen, Ying-Jie, OG

Sheng, Zheng-Ming, OY

Shi, Yuan, $1 \mathrm{Z}$

Si, Lei, $1 \mathrm{~F}$

Song, Enmao, 21

Song, Peng, 1B

Song, Yanrong, IV

Su, Rongtao, OQ, OU

Sun, Ji, 14

Sun, Quan, 1H

Sun, Xiaojie, $1 \mathrm{X}$

Taheri, A., 15

Tang, Binchao, 12

Tang, Xiaojun, OJ

Tian, Jinrong, IV

Uno, Kazuyuki, 1G, IN

Wang, D. N., OR

Wang, Gang, OJ

Wang, Hailin, 22

Wang, Hongyan, $1 \mathrm{H}$

Wang, Jianjun, OT

Wang, Jing, 1B

Wang, Li, IR

Wang, Mu, 20

Wang, Wentao, $0 \mathrm{~J}$

Wang, $\mathrm{Xi}, 17$

Wang, Xiaohao, 12

Wang, Xiaolin, OS, IF, IP

Wang, Yi-bo, 18

Wang, Youwen, $11,1 \mathrm{~J}$
Wang, Yu, 09

Wang, Zefeng, 00

Wang, Zhaokun, ON

Wang, Zhenguo, OF

Wang, Ziwei, ON

Wu, Fenxiang, 10

Wu, Hanshuo, OS

Wu, Jian, OM

Wu, Jianhong, 1D, 1K

Wu, Qipeng, 1 A

Wu, Wenjue, 14

Xiao, Hu, OM

Xie, X. L., Ol

Xing, Ying-bin, 18

$X U$, Jiangmin, $O M$

$X u$, Kun, 14

$X U$, Shanhui, OT

$\mathrm{Xu}$, XiaoJun, $1 \mathrm{H}, 1 \mathrm{M}, 1 \mathrm{P}$

$\mathrm{Xu}, \mathrm{Yi}, 10$

Xu, Zanran, $1 \mathrm{P}$

Yan, Dan, 1T, $1 \mathrm{U}$

Yan, Xingpeng, 17

Yan, Xiongwei, OF

Yan, Yan, $1 R$

Yang, Lijia, OQ

Yang, LV-yun, 18

Yang, Suhui, 09

Yang, XiaoJun, 10

Yang, Zhongmin, OT

Yao, Bao-Quan, OG

Yi, Muyu, IY

Yin, Feifei, 14

You, Kaiming, 11, $1 \mathrm{~J}$

YU, Jian, 1D

Yu, Jinbo, 21

Yu, Linpeng, 10

YU, Quan, 12

Yuan, Xiao, 1Q, 1X, IY

Zeng, Ming, OY

Zha, Hang, ID

Zhang, Feng, 1B

Zhang, Hai-kun, 1B

Zhang, Haiyang, 09

Zhang, Hanwei, OM

Zhang, J. C., Ol

Zhang, Pengfei, $O Q$

Zhang, Shumin, 1T, 1U

Zhang, Tianyu, 19, 1W

Zhang, Xiang, $1 Q, 1 X, 1 Y$

Zhang, Xiangyu, 19, 1W

Zhang, Xiaomin, OF

Zhang, Yu, 06

Zhao, Changming, 09

Zhao, Guomin, 06, 19

Zhao, Jianjun, $1 \mathrm{X}, 1 \mathrm{Y}$

Zhao, Wenguang, 21

Zheng, Jiangang, OF

Zhi, Dong, IF

Zhou, Cheng, 1B

Zhou, J., 0 l 
Zhou, Jun, ON

Zhou, PU, OM, OS, OU, IF, IM

Zhou, Yue, 14

Zhou, Zichao, IP

Zhu, B. Q., 이

Zhu, Changhong, 22

Zhu, Guangzhi, 20, 21, 22

Zhu, Jianqiang, $1 \mathrm{C}$

Zhu, Xiao, 20, 21, 22

Zou, Feng, ON

Zou, Wenlong, 1D

Proc. of SPIE Vol. $100161001601-9$

Downloaded From: https://www.spiedigitallibrary.org/conference-proceedings-of-spie on 26 Apr 2023 Terms of Use: https://www.spiedigitallibrary.org/terms-of-use 
Proc. of SPIE Vol. 10016 1001601-10

Downloaded From: https://www.spiedigitallibrary.org/conference-proceedings-of-spie on 26 Apr 2023 Terms of Use: https://www.spiedigitallibrary.org/terms-of-use 


\title{
Symposium Committees
}

\author{
General Chairs
}

Robert Lieberman, SPIE President, Lumoptix, LLC (United States)

Guangcan Guo, Chinese Optical Society President, University of Science and Technology of China (China)

General Co-chairs

Arthur Chiou, National Yang-Ming University (Taiwan, China)

Jianlin Cao, China Ministry of Science and Technology (China)

Junhao Chu, Shanghai Institute of Technical Physics (China)

Technical Program Chairs

Songlin Zhuang, University of Shanghai for Science and Technology

(China)

Xingde Li, Johns Hopkins University (United States)

Technical Program Co-chairs

Bingkun Zhou, Tsinghua University (China)

Qiming Wang, Institute of Semiconductors (China)

Tianchu Li, National Institute of Metrology (China)

Wei Huang, Nanjing University of Technology (China)

Ying Gu, PLA General Hospital (China)

Huilin Jiang, Changchun University of Science and Technology

(China)

Local Organizing Committee Chair

Qihuang Gong, Peking University (China) 
Local Organizing Committee Co-chairs

Xu Liu, Zhejiang University (China)

Daoyin Yu, Tianjin University (China)

Guoqiang Ni, Beijing Institute of Technology (China)

Shusen Xie, Fujian Normal University (China)

Xiaomin Ren, Beijing University of Posts and Telecommunications

(China)

General Secretary

Yan Li, Chinese Optical Society/Peking University (China)

Local Organizing Committee

Zhiping Zhou, Peking University (China)

Changhe Zhou, Shanghai Institute of Optics and Fine Mechanics, CAS

(China)

Qingming Luo, Huazhong University of Science and Technology

(China)

Chongxiu Yu, Beijing University of Posts and Telecommunication

(China)

Hongda Chen, Institute of Semiconductors (China)

Yongtian Wang, Beijing Institute of Technology (China)

Yiping Cui, Southeast University (China)

Xuping Zhang, Nanjing University (China)

Feijun Song, Daheng Corporation (China)

Cunlin Zhang, Capital Normal University (China)

Yanting Lu, Nanjing University (China)

Yuejin Zhao, Beijing Institute of Technology (China)

Chunqing Gao, Beijing Institute of Technology (China)

Tiegen Liu, Tianjin University (China)

Xiaocong Yuan, Nankai University (China)

Weimin Chen, Chongqing University (China)

Zhongwei Fan, Academy of Optoelectronics (China)

Hanyi Zhang, Tsinghua University (China)

Lan Wu, Zhejiang University (China)

Yongsheng Zhang, University of Science and Technology of China (China)

Hong Yang, Peking University (China)

Xiaoying Li, Tianjin University (China)

Wei Xiong, Chinese Optical Society (China) 


\section{Conference Committee}

Symposium Chairs

Robert A. Lieberman, Lumoptix, LLC (United States)

Wei Xiong, Chinese Optical Society (China)

Conference Chairs

Ruxin Li, Shanghai Institute of Optics and Fine Mechanics (China)

Upendra N. Singh, NASA Langley Research Center (United States)

Robert F. Walter, Schafer Corporation (United States)

Conference Program Committee

Willy L. Bohn, BohnLaser Consult (Germany)

Robert L. Byer, Stanford University (United States)

Dianyuan Fan, Shanghai Institute of Optics and Fine Mechanics (China)

Tomoo Fujioka, Tokai University (Japan)

Mali Gong, Tsinghua University (China)

Shibin Jiang, AdValue Photonics, Inc. (United States)

Do-Kyeong Ko, Gwangju Institute of Science and Technology

(Korea,Republic of)

Zejin Liu, National University of Defense Technology (China)

DeYuan Shen, Fudan University (China)

Yi Su, Institute of Applied Electronics (China)

Shuangchun Wen, Shenzhen University (China)

Zuyan Xu, Technical Institute of Physics and Chemistry (China)

Jianquan Yao, Tianjin University (China)

Tai Hyun Yoon, Korea University (Korea, Republic of)

Jirong Yu, NASA Langley Research Center (United States)

Heping Zeng, East China Normal University (China)

Xiaomin Zhang, China Academy of Engineering Physics (China)

Shouhuan Zhou, North China Research Institute of Electro-optics (China)

Session Chairs

1 Laser Applications

Haiwen Cai, Shanghai Institute of Optics and Fine Mechanics (China)

2 Other Lasers and Applications

Upendra N. Singh, NASA Langley Research Center (United States) 
3 High Power Lasers I

Willy L. Bohn, BohnLaser Consult (Germany)

4 High Power Lasers II

Yan Feng, Shanghai Institute of Optics and Fine Mechanics (China)

5 Fiber Lasers

Yading Guo, Technical Institute of Physics and Chemistry (China)

6 High Field Laser Physics

Baifei Shen, Shanghai Institute of Optics and Fine Mechanics (China)

7 Ultrafast Lasers

Zhigang Zhang, Peking University (China) 